SCIENTIFIC LETTER

\title{
Exercise modulates myocardial protein kinase B/Akt in Zucker obese rats
}

\author{
B Huisamen, A Lochner
}

Heart 2005;91:227-228. doi: 10.1136/hrt.2003.027094

n type 2 diabetes, heart muscle is insulin resistant. Protein kinase $\mathrm{B}(\mathrm{PKB}) / \mathrm{Akt})$ acts as a mediator of the metabolic effects of insulin, including translocation of the insulin sensitive glucose transporter, GLUT4, in a manner dependent on phosphatidylinositol-3-kinase (PI-3-K). ${ }^{1}$ We have previously described dysregulation of $\mathrm{PKB} / \mathrm{Akt}$ in hearts from a rat model of type 2 diabetes, the Zucker fa/fa rat. $^{2}$

Exercise or contraction of heart muscle does not activate PKB/Akt or PI-3-K but can induce GLUT4 translocation and glucose uptake. ${ }^{3}$ However, exercise alleviates peripheral insulin resistance at a currently unknown, post-receptor level. To better understand the mechanism whereby this may be accomplished, we investigated whether chronic exercise will induce beneficial changes in the regulation of PKB/Akt that translates into improved myocardial insulin stimulated glucose uptake.

\section{METHODS}

To accomplish this, we subjected Zucker fa/fa rats (20 weeks old at the beginning of training) and a control group of age matched Wistar rats, to an exercise training programme, and documented changes in glucose uptake, myocardial GLUT4, and $\mathrm{PKB} / \mathrm{Akt}$ expression and phosphorylation, in comparison to their sedentary counterparts. Rats were trained by swimming for four weeks, six days per week, on set times each day, starting at five minutes per day, escalating to 90 minutes per day, to avoid stress. The water temperature was $34^{\circ} \mathrm{C}$ and for half the exercise time, rats were weighted with $2 \%$ of their body weight, carried on their tails. Animals were sacrificed by intraperitoneal injection of sodium pentobarbitone $(0.1 \mathrm{mg} / \mathrm{g})$ after which ventricular myocytes were harvested via standard methods. ${ }^{4}$

2-Deoxy-D-glucose (2-DG) uptake by cardiomyocytes was measured as previously described ${ }^{4}$ while $\mathrm{PKB} / \mathrm{Akt}$ phosphorylation and GLUT4 expression were determined via standard Western blotting techniques and suitable antibodies. PKB/ Akt kinase activity was determined using a commercial nonradioactive assay system (Cell Signaling Technology). Laser scanning densitometry and suitable software (UN-SCAN-IT, Silkscience) were used to analyse the Western blots. Comparisons of datasets were made either by analysis of variance (ANOVA) followed by a Bonferroni correction or a Student's $t$ test for unpaired data. A probability value of $\mathrm{p}<0.05$ was considered significant.

\section{RESULTS}

Blood glucose determinations (Boehringer Mannheim, Accutrend glucose meter) at the time of euthanasia, demonstrated that Zucker fa/fa rats were diabetic at this age (mean (SEM) 10.3 (0.3) mmol/l (fa/fa) $v 7.8$ (0.4) mmol/l (Wistar), $\mathrm{p}<0.01, \mathrm{n}=6$ ). Training lowered the blood glucose of the fa/fa group to $8.8(0.6) \mathrm{mmol} / \mathrm{l} \quad(\mathrm{p}<0.05$, $\mathrm{n}=7$ ).

2-DG accumulation by cardiomyocytes from sedentary diabetic rats (15.3 (1.4) pmol/mg protein/30 mins) was lower

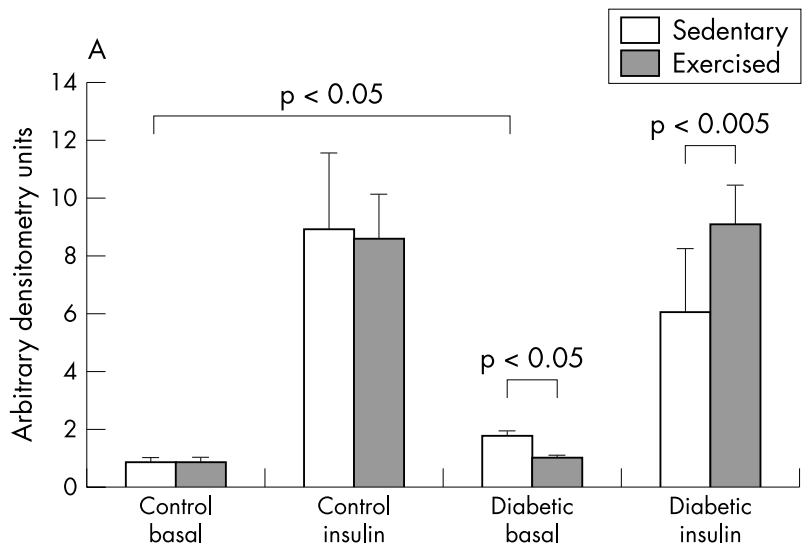

B

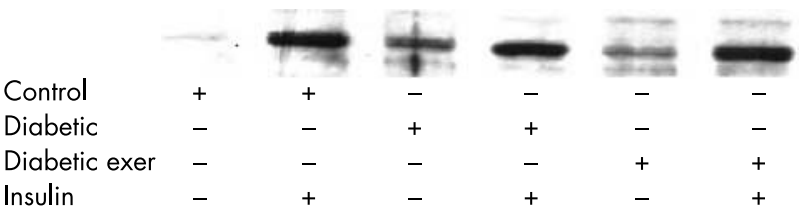

Figure 1 (A) Profile of serine- 473 phosphorylation of PKB/Akt. (B) A representative Western blot of PKB/Akt serine-473 phosphorylation.

than the sedentary control group (25.5 (1.8) pmol/mg protein/30 mins), while insulin could not elicit a significant response in the former $(19.9(3.6) v 99.6(8.7))$. Exercise had no effect on basal glucose uptake (15.2 (1.7) $\mathrm{pmol} / \mathrm{mg}$ protein/30 mins), but improved the insulin stimulated response to $38.5(5.1) \mathrm{pmol} / \mathrm{mg}$ protein $/ 30 \mathrm{mins}$ in the diabetic rats. This was, however, still less than controls after exercise. Concurrently, we found a 30\% lower GLUT4 content in diabetic versus control hearts. Expression of this protein was upregulated by exercise in the diabetic hearts only, to a level similar to that of control hearts after exercise.

We furthermore documented raised serine-473 phosphorylation of $\mathrm{PKB} / \mathrm{Akt}$ in diabetic hearts compared to control hearts, under basal conditions. This was coupled to equal expression and kinase activity of the protein. Nevertheless, it was less sensitive to stimulation by insulin. Following training however, insulin stimulated phosphorylation of $\mathrm{PKB} / \mathrm{Akt}$ was equal to that found in control hearts (fig 1).

\section{DISCUSSION}

It is well established that exercise can improve insulin resistance by sensitising muscle to insulin mediated glucose

Abbreviations: 2-DG, 2-deoxy-D-glucose; PI-3-K, phosphatidylinositol3-kinase; PKB, protein kinase B 
metabolism, and it has been used to treat or prevent type 2 diabetes in patients successfully. Glucose transport is the rate limiting step in muscle glucose utilisation ${ }^{5}$ and the capacity for glucose transport may vary according to the total amount of GLUT4 protein. Exercise raised GLUT4 expression in diabetic hearts; however, despite this upregulation, insulin stimulated glucose uptake was still only $50 \%$ of control values, indicating either defective translocation of the transporter or glucose uptake per se.

A high level of serine-473 phosphorylation of PKB/Akt is usually associated with activation of the kinase and should lead to enhanced glucose uptake. However, we found high basal phosphorylation levels associated with low glucose uptake in sedentary diabetic animals. To the best of our knowledge, this is the first demonstration of such dysregulation at the level of PKB/Akt in diabetes. Exercise normalised the phosphorylation pattern of PKB/Akt in diabetic hearts, an effect probably caused by improved insulin signalling to the applicable phosphatases. This would mean that improved $\mathrm{PKB} / \mathrm{Akt}$ phosphorylation is not the mediator of improved insulin resistance but an effect thereof. Furthermore, despite normal phosphorylation of this kinase in cardiomyocytes from trained diabetic rats and normalisation of GLUT4 expression, insulin stimulated glucose uptake was still curtailed. This result is an important indication that a major alteration in the diabetic heart, leading to faulty glucose metabolism, must be in the functioning or translocation of the GLUT4 protein. These results suggest the possibility that this signalling pathway is a potential target of treatment for type 2 diabetes.

\section{Authors' affiliations}

B Huisamen, A Lochner, Department of Medical Physiology and Biochemistry, Faculty of Medicine, University of Stellenbosch, Republic of South Africa

Correspondence to: Dr Barbara Huisamen, Department of Medical Physiology and Biochemistry, Faculty of Health Sciences, University of Stellenbosch, PO Box 19063, Tygerberg 7505, Republic of South Africa; bh3@sun.ac.za

\section{Accepted 15 April 2004}

\section{REFERENCES}

1 Shepherd PR, Nave BT, Rincon J, et al. Involvement of phosphoinositide 3kinase in insulin stimulation of MAP-kinase and phosphorylation of protein kinase $B$ in human skeletal muscle: Implications for glucose metabolism. Diabetologia 1997;40:1172-7.

2 Huisamen B. Protein kinase B in the diabetic heart. Mol Cell Biochem 2003;249:31-8.

3 Lund S, Pryor PR, stergaard S, et al. Evidence against protein kinase B as a mediator of contraction-induced glucose transport and GLUT4 translocation in rat skeletal muscle. FEBS Letters 1998;425:472-4.

4 Fischer $\mathrm{Y}$, Rosen $\mathrm{H}$, Kammermeier $\mathrm{H}$. Highly insulin-responsive isolated rat heart muscle cells yielded by a modified isolation method. Life Sciences 1991;49:1679-88.

5 Cline GW, Petersen KF, Krssak M, et al. Impaired glucose transport as a cause of decreased insulin-stimulated muscle glycogen synthesis in type 2 diabetes. N Eng J Med 1999;341:240-6.

\section{IMAGES IN CARDIOLOGY}

\section{Value of the unipolar electrogram in the diagnosis of right ventricular perforation following pacemaker implantation}

A 76 year old patient with paroxysmal atrial fibrillation and recurrent syncope caused by sinus arrest was admitted for permanent pacemaker implantation. This was uncomplicated with satisfactory pacing parameters (ventricular lead: threshold $0.7 \mathrm{~V}$ at $0.5 \mathrm{~ms}, \mathrm{R}$ wave $18.1 \mathrm{mV})$. A routine pacing check the following day revealed complete loss of ventricular pacing and abdominal twitching during unipolar ventricular pacing. The sensed unipolar ventricular electrogram demonstrated a dominant, upright $\mathrm{R}$ wave (panel A) and a postero-anterior chest $x$ ray showed the lead tip pointing superiorly, outside the right ventricular boundaries. An epicardial position was suspected and screening in the left anterior oblique projection during subsequent repositioning showed the lead overlying the apical left ventricular free wall (panel B). Repositioning was performed without further complication and a post-procedure unipolar electrogram was obtained (panel C).

Ventricular depolarisation normally occurs from endocardium to epicardium. Endocardial lead positions therefore produce a predominantly negative unipolar electrogram (panel C) and epicardial sites result in a predominantly positive electrogram (panel A).

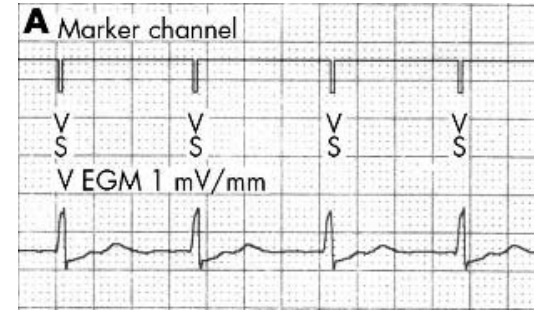

The endocardial electrogram at the repositioned site (panel C) shows a small initial $\mathrm{R}$ wave, which is normal for an apical endocardial right ventricular lead position. This is because during normal intrinsic conduction the earliest site of ventricular activation is the septal aspect of the left ventricular endocardium and septal activation then occurs left to right.

Perforation of the right ventricular free wall during pacemaker implantation is often clinically silent. Findings may include chest pain, a pericardial rub, and a right bundle branch block (RBBB) pacing pattern. If hypotension occurs cardiac tamponade must be excluded.

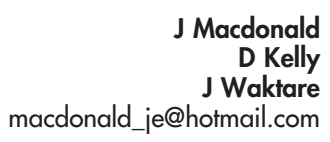

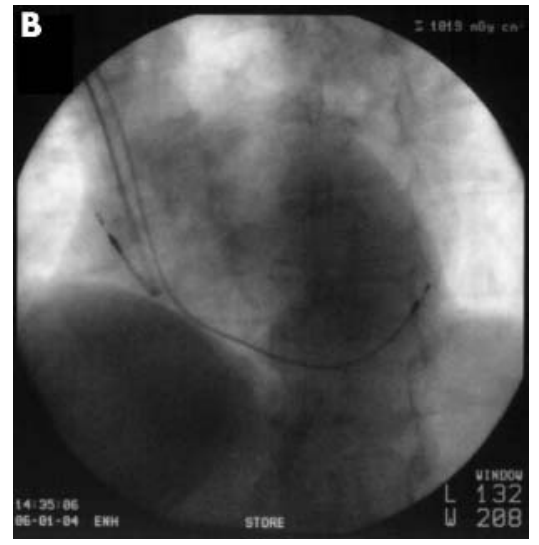

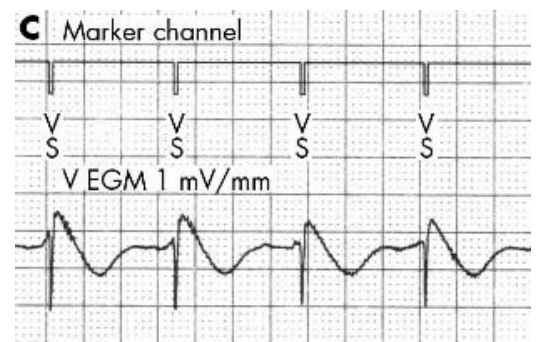

